\title{
Can serial changes of diastolic dysfunction signal incremental risk of chemotherapy- induced heart failure missed by the timing of declining LV ejection fraction?
}

\author{
Ronald G. Schwartz, MD MS, , ${ }^{\mathrm{a} b}$ and Nicholas Venci, $\mathrm{MD}^{\mathrm{a}}$ \\ ${ }^{\text {a }}$ Cardiology Division, Department of Medicine, University of Rochester Medical Center, \\ Rochester, NY \\ ${ }^{\mathrm{b}}$ Nuclear Medicine Division, Department of Imaging Sciences, University of Rochester Medical \\ Center, Rochester, NY
}

Received May 26, 2015; accepted May 26, 2015

doi:10.1007/s12350-015-0194-4

\section{See related article, pp. 824-832}

Cardiologists and oncologists are challenged to optimize safety and effectiveness of chemotherapy. The quest for therapeutic cancer cure is hampered by the risk of cardiotoxicity including potentially fatal clinical heart failure. ${ }^{1}$ Management of chemotherapy administration concordant with strategic baseline EF guideline recommendations for cumulative doxorubicin dose-related timing of accurate and precise radionuclide LVEF measurements, and recommendations for termination of therapy have been demonstrated in high-risk patients to change the natural history of heart failure associated with anthracycline cardiotoxicity. ${ }^{1}{ }^{4}$ Personalizing cardiooncologic care with specific chemotherapy regimens requires use of techniques and monitoring guidelines which have been proven effective by study of long term outcomes in high-risk patients who have abnormal baseline LVEF or those whose residual cancer risk requires consideration of ongoing chemotherapy. ${ }^{4}$ However, evidence of safe and effective management for timing and termination of chemotherapy by serial EF determinations without use of strategic baseline EF guidelines, use of other techniques such as echocardiographic EF and strain imaging, LV and RV volume indices, MIBG, biomarkers,

Reprint requests: Ronald G. Schwartz, MD MS, Cardiology Division, Department of Medicine, University of Rochester Medical Center,

Rochester; ronald_schwartz@urmc.rochester.edu

J Nucl Cardiol 2016;23:833-6.

$1071-3581 / \$ 34.00$

Copyright (C) 2015 American Society of Nuclear Cardiology. and other novel markers remains undefined by prospective trials in high-risk patients receiving anthracycline and other types of chemotherapy. ${ }^{4}$ A potentially important set of novel risk markers of chemotherapy-induced heart failure includes measures of diastolic LV dysfunction which can precede systolic dysfunction in anthracycline and perhaps other chemotherapies and provide a more sensitive and specific predictor of long-term chemotherapy-induced heart failure.

The importance of diastolic dysfunction and its progression to diastolic heart failure, now called "HFpEF", or "'heart failure with preserved ejection fraction", in clinical cardiology, have been recognized for decades. Early studies identified a role for the negative inotropic and chronotropic therapies including the calcium channel blocker verapamil and beta blockers in the management of diastolic heart failure in hypertension or hypertrophic cardiomyopathy. ${ }^{5}{ }^{7}$ Radionuclide ventriculography identified a reduction in the diastolic peak filling rate and prolongation of the time to peak filling associated with HFpEF, and improvements in these dynamic filling characteristics and clinical heart failure with calcium and beta blockade therapy have been demonstrated. ${ }^{5}{ }^{7}$

Diastolic dysfunction was identified by Lee et al. with serial radionuclide angiography as an early marker of doxorubicin cardiotoxicity prior to reduction of LVEF in patients receiving doxorubicin. ${ }^{8}$ This observation was soon followed by reports of early diastolic dysfunction using Doppler echocardiography of LV filling dynamics during doxorubicin treatment. ${ }^{9}$ These observations suggested nearly three decades ago the potential to enhance the diagnostic and prognostic assessment of heart failure risk associated with progressive myocytolysis defined by serial endomyocardial biopsy during anthracycline 
treatment ${ }^{10}{ }^{12}$ prior to the predictive decline of radionuclide LVEF. Recent findings of TOPCAT demonstrating reduced HF admissions and regional variation in the Western but not the Eastern hemispheric countries, despite a negative finding of the primary composite endpoint suggest the potential value of spironolactone as an additional therapy for treatment of chemotherapy-induced HFpEF. ${ }^{13}$

Thus, it would be reasonable to expect serial monitoring of diastolic dysfunction by MUGA parameters of peak filling rate and time to peak filling rate might enhance the sensitivity for detecting cardiotoxicity and lead to recommendations to discontinue therapy earlier than the signal of declining LVEF, enhance the precision of personalized prescription of therapy, and lead to effective treatment of HFpEF with verapamil, beta blockers, and spironolactone. However, evaluation of this enticing hypothesis has eluded our literature and guidelines for decades.

In the current issue of the Journal of Nuclear Cardiology, Reuvekamp and Bulten et al. report a study evaluating diastolic dysfunction as a more sensitive marker of trastuzumab (Herceptin) cardiotoxicity. ${ }^{14}$ Trastuzumab is a humanized monoclonal antibody which targets human epidermal growth factor receptor type 2 (HER2) and has shown to be effective at reducing breast cancer progression, recurrence, mortality, and prolong survival in patients with metastatic breast cancer and in the curative adjuvant setting. ${ }^{15}{ }^{19}$ Trastuzumab has also been shown to increase risk of cardiotoxicity and LV systolic dysfunction. Trastuzamab differs from anthracyclines in the timing of more rapid onset and substantial reversibility of LV systolic dysfunction within a few months of terminating therapy. ${ }^{20}$ Indeed, the investigators of the current study observed trastzumab-induced systolic and diastolic dysfunction by serial MUGA. Importantly, an impairment of MUGA-derived parameters of diastolic dysfunction was not observed prior to systolic dysfunction. The authors conclude serial MUGA measurements of diastolic dysfunction cannot be used as earlier predictors of trastuzumab cardiotoxicity.

Both study limitations and differences in the toxicity of trastuzumab compared to anthracyclines might influence the findings of this study. As always, the methods of patient selection can potentially influence results. Calculation of EF, peaking filling rate (PFR), and time to PFR are key metrics of cardiac function in this study. Of concern, the authors offer no data on the reproducibility of these measurements in their laboratory, although historically high accuracy and reproducibility have characterized radionuclide ventriculographic measurements of LV systolic and diastolic function. Potentially confounding influences of anemia and tachycardia common in cancer patients might have influenced results. A very high proportion of patients (63/77) in this study received anthracyclines, which might have altered the functional response of the LV to trastuzumab. Nevertheless, most patients receiving trastuzumab also receive anthracyclines, so the use of both agents in this study reflects common practice and the need for monitoring this population with advanced breast cancer.

Beyond the potential limitations of this study, what differences in anthracyclines and trastuzumab might account for the absence of diastolic dysfunction preceding systolic dysfunction with trastuzumab cardiotoxicity? Anthracyclines impair cardiac function in a dose-dependent and cumulative manner. Characteristic pathologic features include vacuole formation, myofibrillar disarray, and eventual myocyte necrosis. ${ }^{10}{ }^{12}$ These changes are likely mediated by free radical formation and associated oxidative stress. ${ }^{21}$ In contrast to Type I anthracycline cardiotoxicity with progressive cardiac dysfunction associated with progressive myonecrosis, trastuzumab is now considered to be the prototype agent which produces Type II chemotherapy-related cardiotoxicity which is characterized by reversible cardiac dysfunction not associated with irreversible myocytolysis. ${ }^{20}$ Trastuzumab Type II cardiotoxicity is not dose related, does not occur predictably in all patients, and is reversible within a few months after discontinuation of therapy, and the clinical spectrum of heart failure severity is variable and longterm tolerability is favorable. ${ }^{20,22}$

The mechanism by which trastuzumab exerts its cardiotoxic effects is not clearly understood. HER2 receptors (also known as ErbB2) are expressed by cardiac myocytes and exert cardioprotective effects when neuregulin-1 binds to ErbB3 or ErbB4 causing heterodimerization with ErbB2. Subsequent effects are accomplished via the ERK1/2 and Pl3K/AKT signaling pathways. ${ }^{23,24}$ Animal models have demonstrated that ErbB2 signaling is essential for normal embryonic development. ${ }^{23}$ Furthermore, mice with ventricular restricted ErbB2 deletion developed findings of dilated cardiomyopathy and isolates of cardiac myocytes in affected mice were more susceptible to anthracycline toxicity. ${ }^{24,25}$ Thus, trastuzumab toxicity is believed to be mediated by loss of ErbB2 cardiac repair and survival pathways which normally mitigate the effects of cardiac stress signals, including anthracycline toxicity. ${ }^{26,27}$

The optimal method of monitoring for trastuzumab cardiotoxicity has not yet been clearly identified. The United Kingdom National Cancer Research Institute issued revised recommendations to guide cardiologists and oncologists in monitoring the cardiotoxic effects of trastuzumab. ${ }^{28}$ These guidelines were developed in 
accordance with the cardiac monitoring algorithm in the Herceptin Adjuvant Trial $^{29}$ with additional modifications based on growing clinical experience and understanding of the pathophysiology and natural history of trastuzumab cardiotoxicity. ${ }^{28}$ Prior cardiac disease and anthracycline exposure may potentiate the short-term cardiotoxic effects of trastuzumab, and in the absence of large prospective clinical trials, alternative algorithms for management varying recommendations for close monitoring of these patients have been proposed. ${ }^{4,30}{ }^{32}$ These algorithms continue to focus on serial assessment of LVEF which remains a relatively late marker of cardiotoxicity.

Despite the negative outcome of the trastuzumab trial, Reuvekamp and Bulten must be credited with a broader important question to grace the field: Can serial changes in diastolic dysfunction signal the risk of chemotherapy-induced heart failure missed by the timing of declining LV ejection fraction? If not observed during trastuzumab therapy, can abnormalities of diastolic peak filling rate and time to peak filling defined routinely by planar and SPECT blood pool ventriculography help refine the published guidelines of quantitative radionuclide LVEF which has improved the natural history of anthracycline-induced heart failure? For trastuzumab, it may be the tolerability and survival of its reversible Type II functional cardiotoxicity rests on the accurate identification of the baseline vulnerability of the left ventricle by virtue of systolic or diastolic LV dysfunction and / or LV dilation prior to administration of trastuzumab. In the prior published evaluation of high-risk patients with reduced baseline LVEF prior to or within $100 \mathrm{mg} / \mathrm{m}^{2}$ doxorubin, a substantial percentage of these patients had no clinically identifiable etiology of the baseline LV systolic dysfunction observed; nevertheless with strategic guidelinebased monitoring these patients with baseline LV dysfunction safely received substantial and clinically effective cumulative doses of doxorubicin. ${ }^{3,33}$ Future research in cardio-oncology would likely do well to follow the evidence-based lead of accurate, reproducible, and prognostically validated radionuclide blood pool ventriculography ${ }^{2}-^{4,33,34}$ by identifying the patient with cardiac baseline systolic or diastolic functional vulnerability of the left ventricle who may have reduced tolerance of the substantial, potentially reversible, and unpredictable insult of highly effective trastuzumab in patients with advanced cancer whose cure depends on its safe and timely administration.

Good science leads to many new questions that advance knowledge in the field. The field is indebted to Reuvekamp and Bulten for reminding us radionuclide measures of diastolic dysfunction we have known about for decades may yet have a critical role to play in the assessment of heart failure risk of therapeutic radiation and chemotherapy.

\section{References}

1. Lefrak EA, Pitha J, Rosenheim S, Gottlieb JA. A clinicopathologic analysis of adriamycin cardiotoxicity. Cancer 1973;32:302-14.

2. Alexander J, Dainiak N, Berger HJ, Goldman L, Johnstone D, Reduto L, et al. Serial assessment of doxorubicin cardiotoxicity with quantitative radionuclide angiocardiography. N Engl J Med 1979;300:278-83.

3. Schwartz RG, McKenzie WB, Alexander J, Sager P, D'Souza A, Manatunga A, et al. Congestive heart failure and left ventricular dysfunction complicating doxorubicin therapy. Seven-year experience using serial radionuclide angiocardiography. Am J Med 1987;82:1109-18.

4. Schwartz RG, Jain D, Storozynsky E. Traditional and novel methods to assess and prevent chemotherapy-related cardiac dysfunction noninvasively. J Nucl Cardiol 2013;20:443-64.

5. Bonow RO, Rosing DR, Bacharach SL, Green MV, Kent KM, Lipson RO, et al. Effects of verapamil on left ventricular systolic function and diastolic filling in patients with hypertrophic cardiomyopathy. Circulation 1981;64:787-96.

6. Bonow RO, Udelson JE. Left ventricular diastolic dysfunction as a cause of congestive heart failure. Mechanisms and management. Ann Intern Med 1992;117:502-10.

7. Bonow RO, Dilsizian V, Rosing DR, Maron BJ, Bacharach SL, Green MV. Verapamil-induced improvement in left ventricular diastolic filling and increased exercise tolerance in patients with hypertrophic cardiomyopathy: Short- and long-term effects. Circulation 1985;72:853-64.

8. Lee BH, Goodenday LS, Muswick GJ, Yasnoff WA, Leighton RF, Skeel RT. Alterations in left ventricular diastolic function with doxorubicin therapy. J Am Coll Cardiol 1987;9:184-8.

9. Marchandise B, Schroeder E, Bosly A, Doyen C, Weynants P, Kremer R, et al. Early detection of doxorubicin cardiotoxicity: interest of Doppler echocardiographic analysis of left ventricular filling dynamics. Am Heart J 1989;118:92-8.

10. Billingham ME, Bristow MR. Evaluation of anthracycline cardiotoxicity: Predictive ability and functional correlation of endomyocardial biopsy. Cancer Treat Symp 1984;3:71-6.

11. Billingham ME, Mason JW, Bristow MR, Daniels JR. Anthracycline cardiomyopathy monitored by morphologic changes. Cancer Treat Rep 1978;62:865-72.

12. Mackay B, Ewer MS, Carrasco CH, Benjamin RS. Assessment of anthracycline cardiomyopathy by endomyocardial biopsy. Ultrastruct Pathol 1994;18:203-11.

13. Pfeffer MA, Claggett B, Assman SF, Boineau R, Anand IS, Clausell N, et al. Regional variation in patients and outcomes in the treatment of preserved cardiac function heart failure with an aldosterone antagonist (TOPCAT) trial. Circulation 2015;2014:3442. doi:10.1161/CIRCULATIONAHA.114.013255.

14. Reuvekamp EJ, Bulten BF, Nieuwenhuis AA, Meekes MRA, de Haan AFJ, Tol J, et al. Does diastolic dysfunction precede systolic dysfunction in trastuzumab-induced cardiotoxicity? Assessment with multigated radionuclide angiography (MUGA). J Nucl Cardiol 2015. doi:10.1007/s12350-015-0164-x.

15. Goldhirsch A, Gelber RD, Piccart-Gebhart MJ, de Azambuja E, Procter M, Suter TM, et al. 2 years versus 1 year of adjuvant trastuzumab for HER2-positive breast cancer (HERA): An openlabel, randomised controlled trial. Lancet 2013;382:1021-8.

16. Perez EA, Romond EH, Suman VJ, Jeong JH, Sledge G, Geyer CE $\mathrm{Jr}$, et al. Trastuzumab plus adjuvant chemotherapy for human 
epidermal growth factor receptor 2-positive breast cancer: Planned joint analysis of overall survival from NSABP B-31 and NCCTG N9831. J Clin Oncol 2014;32:3744-52.

17. Slamon DJ, Leyland-Jones B, Shak S, Fuchs H, Paton V, Bajamonde A, et al. Use of chemotherapy plus a monoclonal antibody against HER2 for metastatic breast cancer that overexpresses HER2. N Engl J Med 2001;344:783-92.

18. Smith I, Procter M, Gelber RD, Guillaume S, Feyereislova A, Dowsett M, et al. 2-year follow-up of trastuzumab after adjuvant chemotherapy in HER2-positive breast cancer: A randomised controlled trial. Lancet 2007;369:29-36.

19. Hudis CA. Trastuzumab-mechanism of action and use in clinical practice. N Engl J Med 2007;357:39-51.

20. Ewer MS, Lippman SM. Type II chemotherapy-related cardiac dysfunction: time to recognize a new entity. J Clin Oncol 2005;23:2900-2.

21. Doroshow JH. Effect of anthracycline antibiotics on oxygen radical formation in rat heart. Cancer Res 1983;43:460-72.

22. Guarneri V, Lenihan DJ, Valero V, Durand JB, Broglio K, Hess $\mathrm{KR}$, et al. Long-term cardiac tolerability of trastuzumab in metastatic breast cancer: The M.D. Anderson Cancer Center experience. J Clin Oncol 2006;24:4107-15.

23. Garratt AN, Ozcelik C, Birchmeier C. ErbB2 pathways in heart and neural diseases. Trends Cardiovasc Med 2003;13:80-6.

24. Crone SA, Zhao YY, Fan L, Gu Y, Minamisawa S, Liu Y, et al. ErbB2 is essential in the prevention of dilated cardiomyopathy. Nat Med 2002;8:459-65.

25. Ozcelik C, Erdmann B, Pilz B, Wettschureck N, Britsch S, Hübner $\mathrm{N}$, et al. Conditional mutation of the ErbB2 (HER2) receptor in cardiomyocytes leads to dilated cardiomyopathy. Proc Natl Acad Sci USA 2002;99:8880-5.
26. Chien KR. Herceptin and the heart-a molecular modifier of cardiac failure. N Engl J Med 2006;354:789-90.

27. Perik PJ, de Vries EG, Gietema JA, van der Graaf WT, Smilde TD, Sleijfer DT, et al. Serum HER2 levels are increased in patients with chronic heart failure. Eur J heart Fail 2007;9:173-7.

28. Jones AL, Barlow M, Barrett-Lee PJ, Canney PA, Gilmour IM, Robb SD, et al. Management of cardiac health in trastuzumabtreated patients with breast cancer: Updated United Kingdom National Cancer Research Institute recommendations for monitoring. Br J Cancer 2009;100:684-92.

29. Suter TM, Procter M, van Veldhuisen DJ, Muscholl M, Bergh J, Carlomagno C, et al. Trastuzumab-associated cardiac adverse effects in the herceptin adjuvant trial. J Clin Oncol 2007;25:3859-65.

30. Ewer MS, Vooletich MT, Durand JB, Woods ML, Davis JR, Valero V, et al. Reversibility of trastuzumab-related cardiotoxicity: New insights based on clinical course and response to medical treatment. J Clin Oncol 2005;23:7820-6.

31. Panjrath GS, Jain D. Trastuzumab-induced cardiac dysfunction. Nucl Med Commun 2007;28:69-73.

32. Mackey JR, Clemons M, Cote MA, Delgado D, Dent S, Paterson $\mathrm{A}$, et al. Cardiac management during adjuvant trastuzumab therapy: Recommendations of the Canadian Trastuzumab Working Group. Curr Oncol 2008;15:24-35.

33. Choi BW, Hi B, Schwartz PE, Alexander I, Wackers FJTh, Gottschalk A, et al. Serial radionuclide assessment of doxorubicin cardiotoxicity in cancer patients with abnormal baseline resting left ventricular performance. Am Heart J 1983;106:638-43.

34. Wackers FJ, Berger HJ, Johnstone DE, Goldman L, Reduto LA, Langou RA, et al. Multiple gated cardiac blood pool imaging for left ventricular ejection fraction: Validation of the technique and assessment of variability. AJC 1979;43:1159-66. 